\title{
Evaluations of subjective complexity, pleasingness and interestingness for a series of random polygons varying in complexity
}

HY DAY

ONTARIO INSTITUTE FOR STUDIES IN EDUCATION, UNIVERSITY OF TORONTO

A series of random-shaped polygons varying in number of sides in approximately even logarithmic steps from four to 160 sides was generated. Ss were required to compare all possible pairs of figures on one of three scales-subjective complexity, pleasingness and interestingness. Subjective evaluations of complexity continued to increase with informational content. Pleasingness evaluations described a bimodal function, peaking at the 6-sided and 28-sided levels then falling rapidly with increased complexity. Interestingness evaluations rose to a peak at the 28-sided figure and remained high throughout the rest of the series.

A persisting problem in human motivation is the analysis of the reaction of an individual to stimulus variability. McClelland and others attempted to develop a quantitative theory of affective arousal in which he argued that slight changes in stimulation produce pleasant affect, but that extreme changes are unpleasant (McClelland et al, 1953). Berlyne (1960) suggested that the evaluation of a stimulus may be, at least in part, due to the collative properties of that stimulus, 1.e., its degree of novelty, complexity, incongruity, etc.

It follows, then, that if a subject is presented with a series of stimuli varying in complexity, he should have positive affect toward the less complex patterns but should tend to reject the most complex patterns.

The relevant problems under consideration are of two kinds: the response measure which identifies the affective evaluation of the stimulus and the level of complexity at which positive evaluation gives way to negative evaluation. But an even more primary problem, which has received relatively little consideration, is that of the construction of a set of stimuli varying along a dimension of complexity.

Much of the research into visual complexity to date has tended to rely on stimuli composed of geometrical figures, either as line drawings or solid black-on-white polygons. Attneave and Arnoult (1956) suggested a number of ways of generating random polygons varying in number of sides. They also recommended using an information theory metric of stimulus variability as an objective measure of complexity.

A large number of experimenters adopted the Attneave and Arnoult methods (especially Method 1) to generate shapes which differed in complexity (see
Michels and Zusne, 1965, for review). Of these, Munsinger and Kessen generated the longest series of figures (one of which varied along a continuum in 12 logarithmic steps from five to 40 sides), and published a number of studies in which both children and adults recorded their preferences for figures along this continuum (Munsinger, 1966; Munsinger \& Kessen, 1964, 1966; Munsinger, Kessen, \& Kessen, 1964).

In 1958 Berlyne developed a set of pattern pairs such that the members of each pair differed in level of complexity (Berlyne, 1958). He followed this by a second set of patterns (series $X$ ) which, while of the same basic construction, were composed of a larger number of units; this set, therefore, theoretically had a higher absolute level of complexity than the earlier figures (Berlyne, 1963).

Day (1965b), using 28 of the Berlyne figures, showed that four significantly distinct levels of complexity could be defined. In increasing order of complexity, these levels were: the less complex of the first set (nonX-LC), the more complex of the first set (nonXMC), the less complex of the $X$ set (X-LC), and the more complex of the $\mathrm{X}$ set $(\mathrm{X}-\mathrm{MC})$. He also showed that there is great concordance among Ss in ranking the figures. A number of studies have found increasing exploration and positive affect with increased complexity up to the third level, followed by a de-

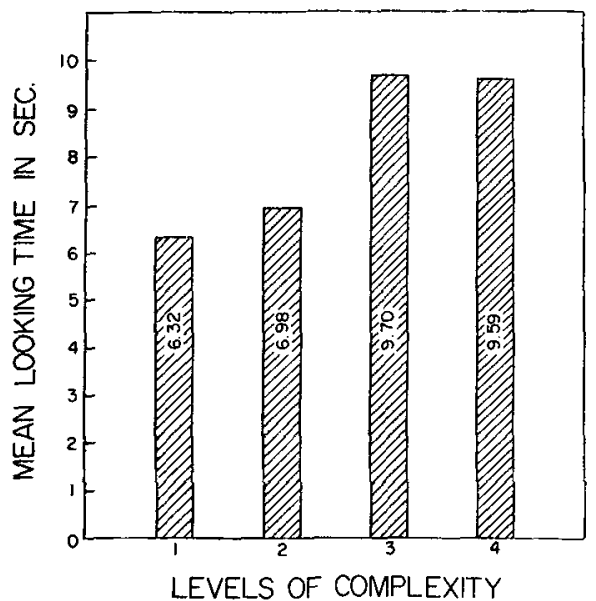

Fig. 1. Distribution of looking time of visual stimulation at different levels of complexity. (from Day, 1966) 


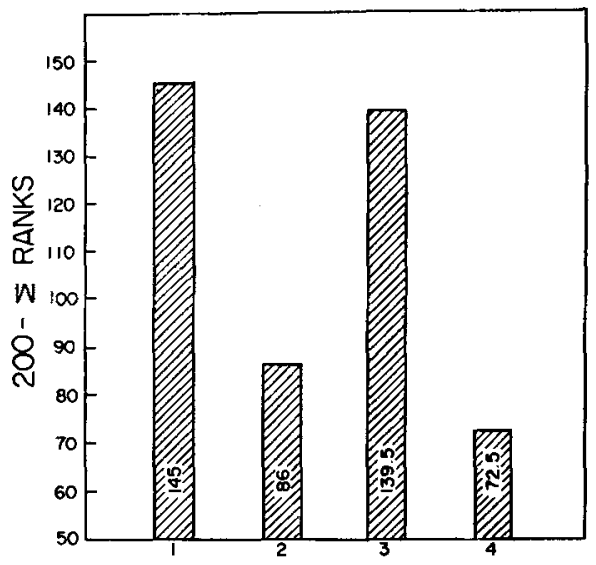

Fig. 2. Evaluation of pleasingness rankings for visual stimuli of different levels of complexity. (from Day, 1965b)

crease or flattening at the fourth level. This is exemplified in Fig. 1 by the results of one study on the relationship of looking time to complexity (Day, 1966).

Berlyne (1963) introduced verbal evaluations of "interestingness" and "pleasingness," and showed that they represented quite different attitudes towards complexity. The former, interestingness, generally reflected a higher evaluation of MC alternatives, while the latter, pleasingness, reflected a higher evaluation of the LC alternatives. Day, working with 28 Berlyne figures (Day, 1965b), found that interestingness rankings rose to peak at the third level of complexity, while pleasingness rankings were apparently influenced by factors other than complexity. These distributions are llustrated in Figs. 2 and 3.

The bimodal function in Fig. 3 may possibly be due to the effect of symmetry, which was considered by Berlyne to be a factor of complexity, and was clearly greatest in the first and third levels of complexity.

Munsinger and Kessen used a series of random patterns in a test of paired-comparisons and found a W-shaped function of preference over complexity (Munsinger \& Kessen, 1964). They attributed the peaks at the extremes of the distribution to "meaningfulness"' of the material and other causes. Eliminating the effects of these factors, Munsinger and Kessen were able to reduce the shape to an inverted U function corresponding to Fechner's results. Terwilliger (1963), on the other hand, used a seven-point rating scale and found that his data fitted the adaptation level hypothesis of a sharp dip in the middle of the distribution flanked by sharp rises on either side. More recently, Looft presented some of the Munsinger and Kessen figures in all possible triads, and had Ss rank the members of each triad. Analysing each S's scores separately, he found large individual differences and reported that $50 \%$ of the $\mathrm{Ss}$ had bi- modal functions while only $21 \%$ had unimodal functions (Looft, 1966).

Following Munsinger and Kessen (1964), it was decided in this series of studies to adopt the information theory metric of stimulus variability and assume a positive monotonic relation between the number of sides in the stimulus and the amount of information in it. Specifically, a series of patterns was so designed as to vary in approximately logarithmic steps from a four to a 160-sided figure. 1

A series of four experiments was performed, in which verbal evaluations of solid polygons varying from four to 160 sides were examined. The verbal responses were those of "complexity," "interestingness," and "pleasingness."

\section{Stimulus Material}

\section{GENERAL METHOD}

The figures were black-on-white solid polygons differing in number of sides. The method of construction was adapted from Attneave and Arnoult (1956) Method 1, using graph paper and a random numbers table. The size of all the figures was equated, the figures were photographed on $35 \mathrm{~mm}$ high contrast film, and projected side by side as black-on-white images. The size of the figures was limited only by the size of the screens available.

\section{Procedure}

The paired-comparison method was used so that every possible combination of figures was displayed. Each pair was presented for a 3-sec. exposure followed by 3 sec. of no-display to allow the recording of the response. The order of presentation of each pair was randomly chosen, as was the side on which each alternative was displayed.

All $\mathrm{Ss}$ in each study participated in a single group session. Approximately one-third were instructed to mark the More Complex, one-third the More Inter-

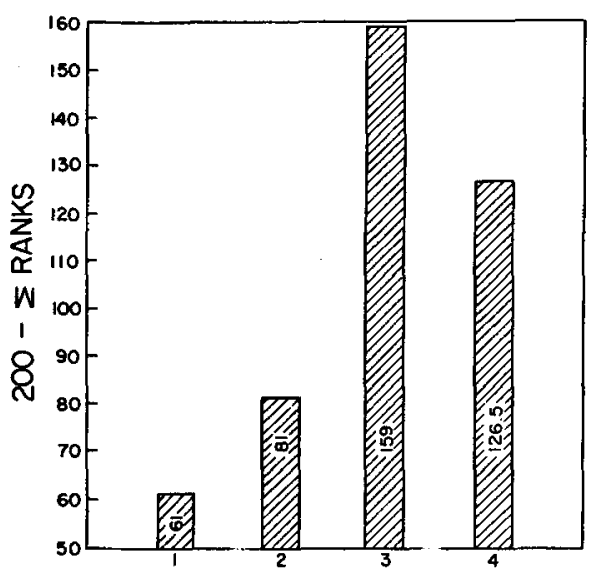

Fig. 3. Evaluation of interestingness rankings for verbal stimuli at different levels of complexity. (from Day, 1965b) 


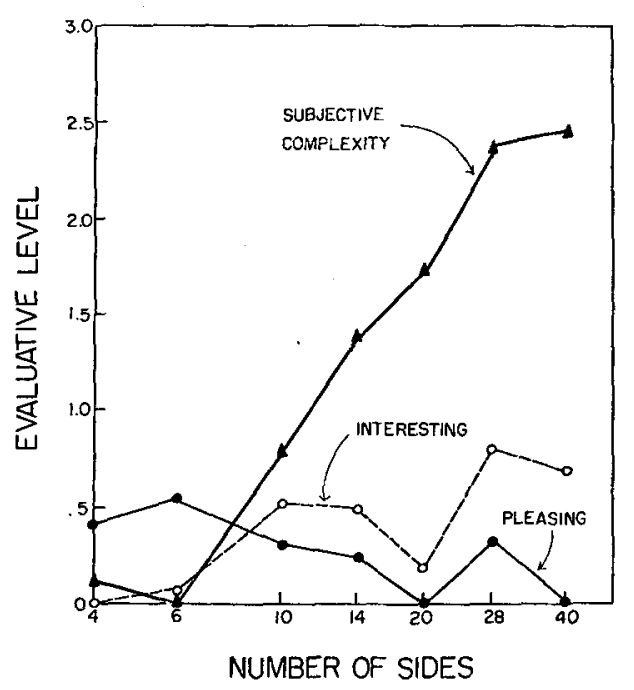

Fig. 4. Subject evaluations of figures differing in numbers of sides placed on a log metric. (Study 1)

esting, and one-third the More Plzasing alternatives of each pair of slides. Answer booklets were distributed so that Ss sat between Ss using different response measures.

Thurstone's Case $V$ method of attitude scaling was used to analyze the paired-comparisons data, substituting a level of complexity for an attitudinal statement. The analysis recommended by Edwards (1957) was followed. This analysis allows one to obtain the frequency with which each stimulus item is judged to be more favorable than every other stimulus item. The matrix of frequency scores is converted into a matrix of probability scores and then into one of $\mathrm{Z}$ scores. The $\mathrm{Z}$ scores in each column are summed and the means obtained. The means of the $Z$ values in every column, therefore, express the scale value of that particular stimulus in terms of its deviation from the mean of all the scale values. By adding a constant to each of these scores the distribution can be adjusted so that the lowest score will be equal to zero and the others relative to that score. It should be noted that predictions about absolute differences among the various curves are meaningless, and only the shapes of the distributions are to be constdered.

\section{STUDY 1}

The stimulus slides were a series of seven polygons varying in approximately even logarithmic steps from four to 40 sides. The Ss were 245 students in an extension Introductory Social Science course at York University, Toronto.

\section{Resulis}

The results of the subjective evaluations of complexity, interestingness, and pleasingness are presented in Fig. 4.
Subjective evaluation of complexity was found to increase with the amount of variability in the figures. Two deviations from linearity appeared, once where Ss seemed to feel that the 6-sided figure was less complex than the 4-sided figure, and once where the 40 -sided figure was seen as equally complex to the 28-sided figure.

The distribution of interestingness scores generally appeared to rise, with fluctuations, to the 28-sided figure level and then to drop very slightly. On the other hand, pleasingness evaluations seemed to be distributed somewhat randomly, except that they tended to decrease with increasing complexity. The 20-sided figure appeared to be extremely poor in eliciting evaluations of interestingness and pleasingness.

Flgure 5 shows the distribution of interestingness and pleasingness evaluations of the figures when they are ordered along a dimension of subjective evaluation of complexity. Since subjective complexity was almost linearly distributed, differences from this figure to Fig. 4 are minimal. However, this figure presents the relationships among the response variables and, as such, is similar to a correlational distribution.

\section{STUDY 2}

The finding that interestingness reached a peak at the 28-sided figure level and then decreased slightly was suggestive of earlier work by this author (see Fig. 3). It might be that with even more complex stimulation interest would continue to decrease. The next step was thus to extend the series in order to examine the effects of additional stimulus complexity on all three evaluations.

It was decided to increase the scope of the stimulus materlal both longitudinally and laterally, extending the series to 90-sided figures with the addition of three more figures, and doubling the number of slides at each level by using inverted figures $\left(180^{\circ}\right.$

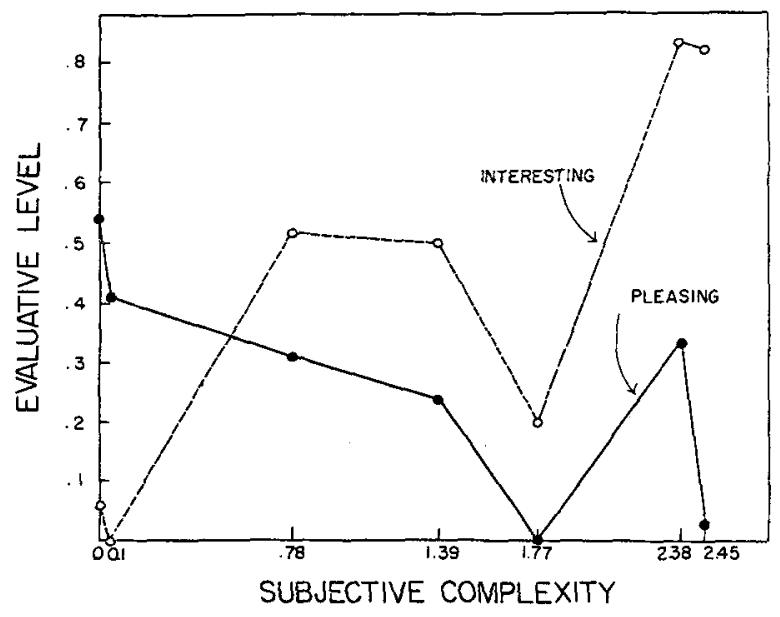

Fig. 5. Subjective evaluations of figures varying in subjective complexity. (Study 1) 


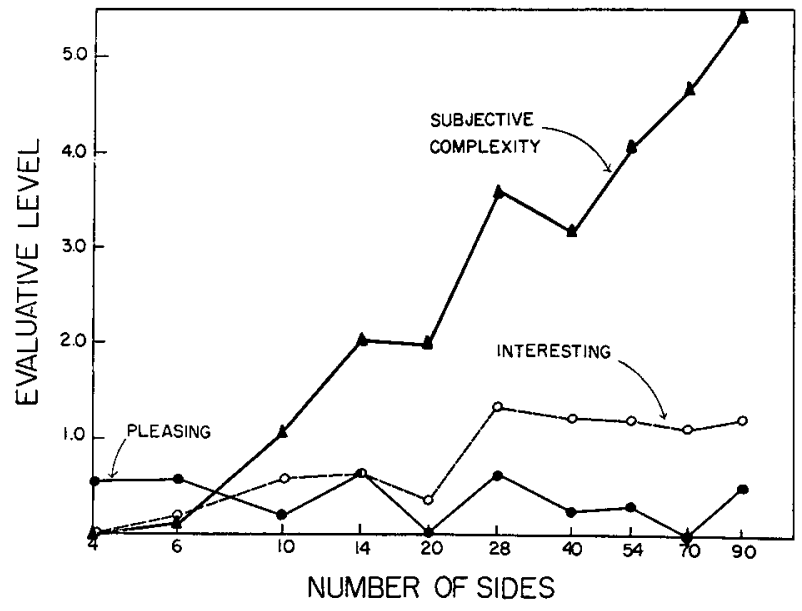

Fig. 6. Subjective evaluations of figures varying in levels of informational content. (Study 2)

rotation plus inversion). This formed a set of 20 figures and a total of 190 paired comparisons. The Ss were 64 freshman students in an introductory psychology course at the University of Toronto. They participated in one group, approximately evenly divided into the three response categories. The order of presentation of slides was randomized as before.

\section{Results}

No differences were found between evaluations of the figures and their inverted rotations. Each pair of figures thus actually appeared four times in the series, and the data for the four pairs were combined in the analysis. Figure 6 shows the distribution of evaluation scores for the polygons.

An examination of subjective complexity evaluations in Fig. 6 suggests that the plateau at the 40-sided level which had been found in Study 1 is a peculiarity of that particular figure, for subjective evaluation of complexity continued to rise with informational content. The evaluation of interestingness appeared to reach a peak at the 28-sided figure level and then to drop somewhat with added variability. Pleasingness, on the other hand, vacillated greatly and appeared to be affected by factors other than complexity.

\section{STUDY 3}

The findings of the exaggerated valleys in all three evaluations of the 20-sided pattern posed a problem. The adaptation level hypothesis would have predicted the valley at approximately this level but not for all response variables. Moreover, the valleys had appeared in Study 1, when the 20-sided figure was not at the mean of the distribution.

It was therefore decided to replicate Study 2, substituting another 20-sided figure and changing the order of presentations. It was hoped in this way to

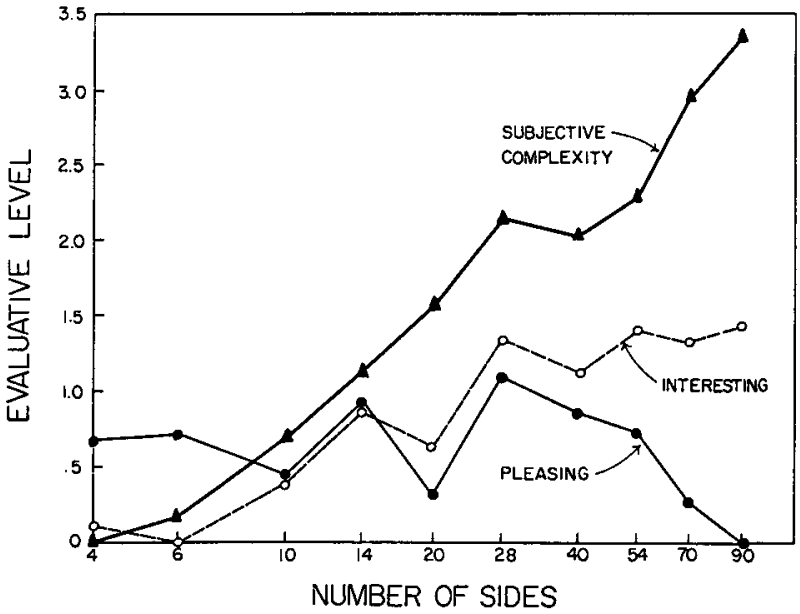

Fig. 7. Subjective evaluations of figures varying in levels of informational content. (Study 3)

determine if the deviation was caused by properties of the pattern or by virtue of that figure's location in the series.

The Ss were 117 female students at Ryerson Institute of Technology in Toronto.

\section{Results}

The results of this study are presented in Fig. 7. After replacing the 20-sided figure, the dip in the subjective complexity distribution disappeared. However, evaluations of interestingness and pleasingness continued to remain unusually low at the level of the 20-sided figure. Aside from other minor variations, evaluation of interestingness again increased from 4-sided to 28-sided figures and appeared to reach some sort of asymptote. Pleasingness, on the other hand, fluctuated wildly, but appeared to decrease from the 28- to 90-sided level in this study. While some of these variations might possibly be due to subject differences (Ss in the present study were 117 female students in an institute of technology as compared to a mixed class of $64 \mathrm{Ss}$ in an honor course in psychology at a university), it is also likely that since the paired-comparisons method was used, change in affect towards one figure influences score values for all the other figures. Generally, the three studies were consistent in finding a valley at the 20-sided level and a peak at the 28-sided level for both pleasingness and interestingness.

\section{STUDY 4}

Four questions have now become apparent, the answers to which depend upon extending the stimulus material into still higher levels of variability. (a) Does the subjective evaluation of complexity continue to increase with an increasing number of sides, or does it eventually reach a peak and begin to drop, as sug- 
gested by Heckhausen (1964)? (b) Does the distribution of interestingness scores reach an asymptote at the 28-sided level and remain there, or does it decrease with increasing complexity? (c) Does the pleasingness distribution continue to fluctuate randomly with complexity, or is there a definitive trend towards a decrease in pleasingness with increasing complexity? (d) Since the valley at the 20-sided figure level did not disappear with the substitution, there is reason to believe that it is at the adaptation level. Would the extension of the series in an upward direction that displaces the point of depression also move the valley upwards?

Since it had been found that 190 comparisons were the maximum that a group could make competently, it was decided to limit the series to 20 figures. A series of patterns in 20 logarithmic steps from 10 to 160 sides was therefore constructed. The Ss were 575 students, mostly females, from a local teacher's college. Of these, 179 compared the stimuli for complexity, 182 for pleasingness, and 214 for interestingness.

\section{Results}

The results of this study are displayed in Fig. 8 . With minor variations, subjective responses of complexity continued to rise with objective variabillty, thus confirming earlier findings by Day (1965a) and the theoretical position held by Berlyne (1960). Yet it seems that at the most complex levels, discrimination of complexity among figures becomes more difficult, and an asymptote of subjective complexity is approached.

The distributions of both interestingness and pleas-

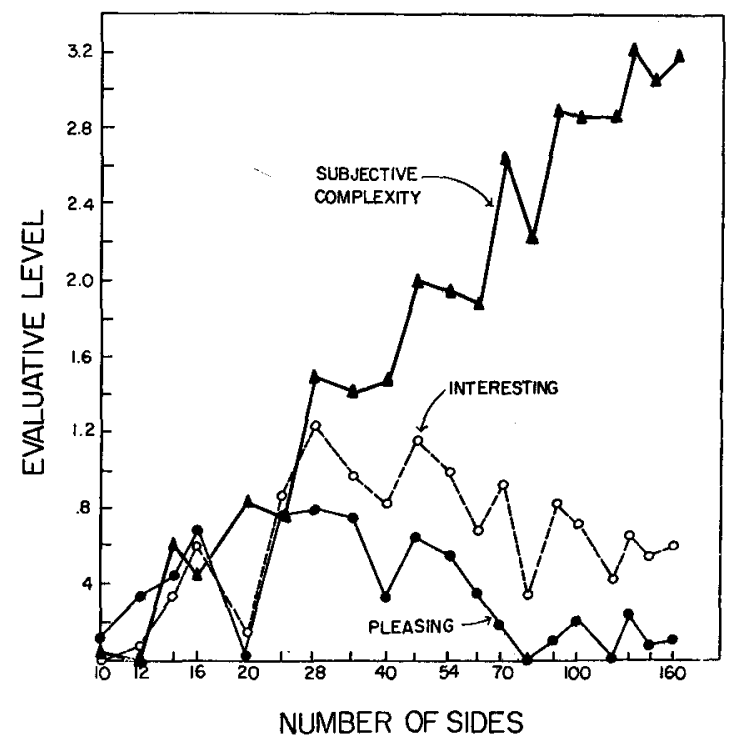

Fig. 8. Subjective evaluations of figures varying in levels of informational content. (Study 4)

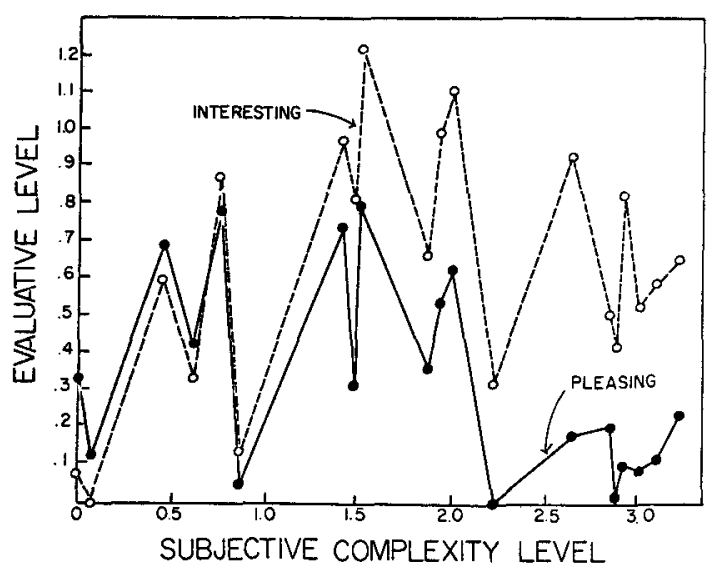

Fig. 9. Subjective evaluations of figures varying in subjective levels of complexity.

ingness evaluations over the range of variability from 10 to 160 sides reach a peak at the 28-sided level of complexity. However, where pleasingness drops off sharply with increasing complexity, interest in high levels of complexity appears to be sustained more strongly. This is demonstrated again in Fig. 9, which shows the distribution of interestingness and pleasingness scores along the dimension of subjective complexdty.

\section{DISCUSSION}

Despite the minute differences in complexity between neighboring figures, the use of only one slide at each level may have exaggerated the effect of random fluctuations, and thus the points at which interest and pleasingness peak may be disguised by random variability. Pleasingness evaluations appear to fluctuate greatly and to be correlated only slightly with complexity. If the data from the four studies are combined, and the valleys at the 20 -sided level are attributed to peculiarities of the figures, 2 the distribution of pleasingness evaluations seems to be bimodal, peaking once at the 6-sided level and again at the 28-sided level. These results are consistent with earlier results by this author, who found a bimodal distribution of pleasingness over complexity and a U-shaped distribution of pleasingness over interestingness (Day, 1965b). In the present series of studies too, pleasingness was high when interest was low (6-sided level) and again when interest was at its peak (28-sided level).

The adaptation level hypothesis predicting a shift in the valley in the fourth study was not confirmed. The deepest valleys remained at the 20-sided level, while the lesser depressions at the 40-sided level have been manifest in all four studies; these cannot be attributed to their location in the series. Coincidentally, depressions recurred at the levels of the 10-sided, 20-sided, 40-sided, 80-sided, and 122- 
sided figures. Whether there is any significance to this remains to be seen in future replications.

The results of these studies suggest that the use of the response measures of preference and likedon't like by other researchers may result in confusion of feelings of interest and pleasure in the stimull. A yet unpublished study by this author found that Ss, looking at polygons "as long as you like," took the same length of time as Ss looking "as long as the flgures are interesting," and significantly longer than when instructed to look "as long as the figures are pleasing." Berlyne has suggested that verbal responses of pleasingness reflect a rousalreducing stimulus properties, while responses of interestingness reflect internal processes closely related to arousal-raising stimulus properties (Berlyne, 1963). This is confirmed by the results of the present studies. Pleasingness appears to be high for low levels of complexity but low at extremely high levels of complexdty. Interest seems to increase with complexity to a peak and to remain fairly high with additional complexity.

\section{References}

Attneave, F., \& Amoult, M. The quantitative study of shape and pattern perception. Psychol. Bull., 1956, 53, 452-471.

Berlyne, D. E. The influence of complexity and novelty in visual figures on orienting responses. J. exp. Psychol., 1958, 55, 289-296.

Berlyne, D. E. Conflict arousal and curiosity. New York: McGrawHill, 1960.

Berlyne, D. E. Complexity and incongruity variables as determinants of exploratory choice and evaluative ratings. Canad. $J$. Psychol., 1963, 17, 274-290.

Day, H. Brief note on the Berlyne-Heckhausen controversy. Psychol. Rep., 1965a, 17, 225-226.

Day, H. Exploratory behavior as a function of individual differences and level of arousal. Ph.D. thesis, University of Toronto, 1965b,
Microfilm \#66-1064, University Microfilms Inc., Ann Arbor, Michigan.

Day, H. Looking time as a function of stimulus variables and individual differences. Percept. mot. Skills, 1966, 22, 423-428.

Edwards, A. L. Techniques of attitude scale constructions. New York: Appleton-Century-Crofts, 1957.

Heckhausen, H. Complexity in perception: phenomenal criteria and information theoretic calculus-A note on D. E. Berlyne's "Complexity effects". Canud. J. Psychol., 1964, 18, 168-173.

Loott, W. R. An investigation of the preference function for visual random shape patterns. Paper presented at the Midwestern Psychological Association annual meeting, May, 1966.

Mcclelland, D. C., Atkinson, J. W., Clark, R. A., \& Lowell, E. L. The achievement motive. New York: Appleton-Century-Crofts, 1953.

Michels, K. M., \& Zusne, L. Metrics of visual form. Psychol. Bull., 1965.

Munsinger, H. Multivariate analysis of preference for variability. J. exp. Psychol., 1966, 71, 889-895.

Munsinger, H., \& Kessen, W. Uncertainty, structure and preference. Psychol. Monogr., 1964, 78, No. 9 (Whole No. 586).

Munsinger. H., Kessen, W. Stimulus variability and cognitive change. Psychol. Rev., 1966, 73, 164-178.

Munsinger, H., Kessen, W., \& Kessen, Marion L. Age and uncertainty: Developmental variations in preference for variability. J. exp. child Psychol., 1964, 1, 1-15.

Terwilliger, R. R. Patterns complexity and affective arousal. Percept. mot. Skills, 1963, 17, 387-395.

\section{Notes}

1. Prints of the figures used in these studies are available from the author on request.

2. Since this paper was written a study was completed which included a comparison of the two 20-sided figures used in these studies with six others. The results indeed showed that these two figures were considered to be less pleasing and interesting but no less complex than the other six 20 -sided figures.

3. This research was supported in part by Ontario Mental Health Foundation Grant \#70 and National Research Council Grant \#APT73 to Dr. D. E. Berlyne, University of Toronta.

(Accepted for publication March 20.1967.) 